\title{
Modélisation géometrique du filetage à la fraise
}

\author{
Guillaume Fromentin $^{1, a}$, Anna Carla Araujo ${ }^{2}$, Gérard Poulachon ${ }^{1}$ \\ ET YANN PAIRE ${ }^{1}$ \\ 1 LaBoMaP, Arts et Métiers ParisTech, 71250 Cluny, France \\ 2 Université Fédérale de Rio de Janeiro, Brésil
}

Reçu le 26 novembre 2010, accepté le 10 octobre 2011

\begin{abstract}
Résumé - Les opérations de fraisage sont relativement complexes à mettre au point car il y a de nombreux paramètres de coupe et caractéristiques géométriques de l'outil. De plus, les efforts de coupe varient en usinage, du fait de la discontinuité de la coupe. La modélisation des opérations de fraisage et la détermination des efforts de coupe devraient permettre d'évaluer, rapidement et avec peu d'essais, l'effet de paramètres géométriques de l'outil et des conditions de coupe, et définir ainsi un domaine d'utilisation acceptable. Toutefois pour réaliser cet objectif, il est nécessaire de disposer de modèles robustes et capables de reproduire l'effet de différents paramètres, en particulier ceux liés à la géométrie de l'outil. Le filetage à la fraise est une technique qui permet de générer aussi bien des filets intérieurs qu'extérieurs par interpolation hélicoïdale avec un outil ayant le profil du filet. Cette technique possède plusieurs avantages, notamment par rapport au taraudage, et s'avère adaptée au contexte de production de pièces à forte valeur ajoutée. D'un point de vue géométrique, le filetage à la fraise est une configuration d'usinage 3D complexe, et ceci par la trajectoire, la géométrie de coupe de l'outil et la section coupée. L'étude proposée traite de la modélisation géométrique du filetage à la fraise qui est une étape préliminaire indispensable notamment à la modélisation des efforts de coupe. L'approche développée expose une formulation analytique complète de la géométrie de la fraise (arête de coupe, face de coupe, face en dépouille) et examine les spécificités de ce type d'outil en terme de variation d'angle d'outil. Par ailleurs, elle propose une formulation simplifiée de la section coupée.
\end{abstract}

Mots clés : Filetage à la fraise / géométrie de coupe / angle de coupe / épaisseur coupée

\begin{abstract}
Geometrical modelisation of thread milling process. Milling operation parameters, such as cutting and geometrical parameters, are quite difficult to define because of the variation of chip load during the process. Modeling the cutting forces is an efficient help to evaluate the effects of each parameter with the support of a few number of machining tests, and in order to define the available parameters domain. Nevertheless, the modeling needs improvement to be more precise and consequently be able to compare the effect of changing parameters as tool geometry. Thread milling is a machining technique which can produce both internal and external threads with using a machine tool, a helicoidal interpolation and a tool having the thread profile. This technique has several advantages compared to cut tapping and it is well adapted to the production of high cost parts. From a geometric point of view, thread milling is a quite complex 3D machining problem, due to the tool center trajectory, cutting mill geometry and cutting section. The present study deals with the geometric modeling of thread milling as a preliminary step for cutting force modeling. The developed approach is based on the complete analytical formulation of the mill geometry (cutting edge, rake face, clearance face). A simplified formulation for uncut chip thickness is established and the specificities of some parameters are examined correlating to the tool angle variation.
\end{abstract}

Key words: Thread milling / cutting geometry / tool angles / uncut chip thickness

\footnotetext{
${ }^{a}$ Auteur pour correspondance : guillaume.fromentin@ensam.eu
} 


\section{Nomenclature}

\begin{tabular}{|c|c|}
\hline \multicolumn{2}{|r|}{ Indices et abréviations } \\
\hline $\mathrm{f}$ & relatif à la fraise \\
\hline acf & arête de coupe frontale \\
\hline aci & arête de coupe inférieure \\
\hline acs & arête de coupe supérieure \\
\hline \multicolumn{2}{|r|}{ Référentiels et paramètres } \\
\hline$\left(o, \mathbf{e}_{1}, \mathbf{e}_{2}, \mathbf{e}_{3}\right)$ & repère lié à la fraise \\
\hline$\left(O, \mathbf{E}_{1}, \mathbf{E}_{2}, \mathbf{E}_{3}\right)$ & repère lié à la pièce \\
\hline$a_{z}$ & altitude d'un point de l'arête dans repère outil (mm) \\
\hline$r, \theta, z$ & coordonnées cylindriques \\
\hline$\theta$ & position angulaire de la fraise dans le repère de la pièce \\
\hline \multicolumn{2}{|r|}{ Dimensions du filet } \\
\hline$D$ & diamètre nominal (mm) \\
\hline$D_{1}$ & diamètre intérieur (mm) \\
\hline$D_{2}$ & diamètre sur flancs (mm) \\
\hline$P$ & pas (mm) \\
\hline$p$ & pas angulaire $\left(\mathrm{mm} \cdot \mathrm{rad}^{-1}\right)$ \\
\hline \multicolumn{2}{|r|}{ Caractéristiques de la fraise } \\
\hline$\overline{D_{\mathrm{f}}}$ & diamètre extérieur ( $\mathrm{mm})$ \\
\hline$D_{2 \mathrm{f}}$ & diamètre sur flancs (mm) \\
\hline$R_{\mathrm{FD}}$ & rayon de la face de dépouille $(\mathrm{mm})$ \\
\hline$Z$ & nombre de dents \\
\hline$\alpha_{\mathrm{ff}}$ & angle de dépouille en travail de la fraise sur acf $\left(^{\circ}\right)$ \\
\hline$\gamma_{\text {of }}$ & angle de coupe orthogonal de la fraise sur acf $\left(^{\circ}\right)$ \\
\hline$\lambda_{\mathrm{sf}}$ & angle des goujures (angle d'hélice) sur le diamètre $D_{\mathrm{f}}\left({ }^{\circ}\right)$ \\
\hline$\theta_{\mathrm{ac}}\left(a_{z}\right)$ & position angulaire d'un point de l'arête dans le repère de la fraise \\
\hline \multicolumn{2}{|r|}{ Angle d'outil } \\
\hline $\mathbf{P}_{\mathrm{n}}$ & plan normal à l'arête \\
\hline $\mathbf{P}_{\mathrm{f}}$ & plan de travail \\
\hline $\mathbf{P}_{\mathrm{o}}$ & plan orthogonal de l'outil \\
\hline$\alpha_{\mathrm{o}}$ & angle de dépouille orthogonal local $\left(^{\circ}\right)$ \\
\hline$\gamma_{\mathrm{n}}$ & angle de coupe normal local $\left(^{\circ}\right)$ \\
\hline \multicolumn{2}{|r|}{ Paramètres de coupe et cinématiques } \\
\hline$f_{z}$ & avance par dent $\left(\right.$ mm.tour ${ }^{-1} \cdot$ dent $\left.^{-1}\right)$ \\
\hline$f_{z 12}$ & avance projetée dans le plan $\left(\mathbf{E}_{1}, \mathbf{E}_{2}\right)\left(\mathrm{mm} \cdot \operatorname{tour}^{-1}\right.$.dent $\left.{ }^{-1}\right)$ \\
\hline$f_{z 3}$ & avance axiale $\left(\mathrm{mm} \cdot\right.$ tour $^{-1} \cdot$ dent $\left.^{-1}\right)$ \\
\hline$\Omega$ & vitesse angulaire du centre fraise $\left(\mathrm{rad} . \mathrm{s}^{-1}\right)$ \\
\hline$\omega$ & vitesse de rotation de la fraise $\left(\mathrm{rad} . \mathrm{s}^{-1}\right)$ \\
\hline \multicolumn{2}{|r|}{ Objets géométriques } \\
\hline $\mathbf{A C}\left(a_{z}\right)$ & arête de coupe \\
\hline $\operatorname{CFD}\left(a_{z}\right)$ & centre d'une section de la face de dépouille \\
\hline $\mathbf{F C}\left(r, a_{z}\right)$ & face de coupe \\
\hline $\mathbf{F D}\left(a_{z}, \theta\right)$ & face de dépouille \\
\hline $\mathbf{V R}\left(a_{z}\right)$ & vecteur rayon passant par l'arête \\
\hline $\operatorname{VNR}\left(a_{z}\right)$ & vecteur normal à VR \\
\hline $\operatorname{PF}\left(a_{z}\right)$ & profil de la fraise \\
\hline $\mathbf{P}_{f i}$ & $i^{\text {ème }}$ point du profil de la fraise \\
\hline $\mathbf{T C F}(\mathrm{t})$ & trajectoire du centre de la fraise \\
\hline
\end{tabular}

\section{Introduction}

Il existe une multitude de types de fraise, de technologies d'outil, de stratégies de fraisage. Le fraisage est une technique de coupe 3D où les conditions de coupe sont au nombre de quatre (vitesse de coupe, avance, engagement axial et radial). De plus, la section coupée est variable au cours du temps et souvent aussi le long de l'arête. Face à cette complexité, il est utile de disposer de modèles d'efforts de coupe permettant de prédéterminer l'effet de la géométrie d'outil et des conditions de coupe.

L'étude ci-dessous traite de la modélisation géométrique de la coupe lors d'une opération de filetage à la fraise, étape nécessaire pour ensuite calculer les efforts de coupe. Les outils de modélisation développés dans ce cas présentent un intérêt direct pour cette technique et sont, de plus, applicables à d'autres situations de fraisage. 


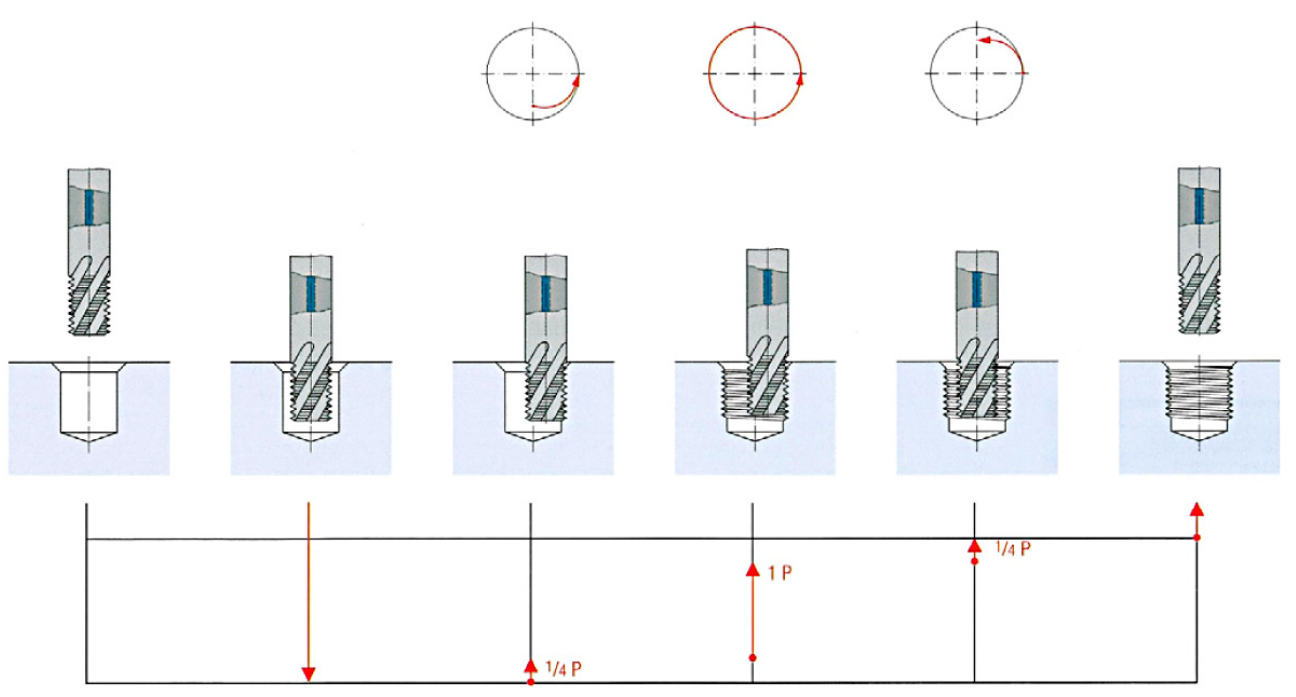

Fig. 1. Cycle de filetage intérieur, en une passe et en avalant, à la fraise (source : fabricant Emuge Catalogue 130).

Le filetage à la fraise est une opération d'usinage avec une fraise de forme, ayant un profil semblable au filet qui doit être usiné. Il existe diverses technologies d'outil et l'étude proposée traite du cas de l'obtention de filet métrique avec des outils ayant un profil composé de plusieurs pas comme les fraises monoblocs. Un cycle de filetage intérieur en une passe à la fraise se déroule comme indiqué sur la figure 1. Après engagement de la fraise, l'outil a une trajectoire hélicoïdale sur un pas afin de générer le filet. Dans le cas d'un usinage en deux ou trois passes, le rayon de l'interpolation hélicoïdale augmente à chaque passe.

Le filetage à la fraise est une technique qui permet de réaliser divers profils de filets, aussi bien intérieurs et qu'extérieurs. Une même fraise peut usiner des filets à gauche et à droite, de différents diamètres mais de même pas, en avalant ou en opposition. Cette technique est donc plus flexible que le taraudage, et concurrence ce dernier. En cas de casse de la fraise, l'outil peut être retiré car il est d'un diamètre inférieur au filet. Cela permet de reprendre l'opération de fraisage sans rebuter la pièce. De plus, le fraisage permet d'avoir des vitesses de coupe adaptées alors qu'en taraudage, l'inversion du sens de rotation de la broche est contraignante sur ce point. Enfin, le filetage à la fraise réduit, par rapport au taraudage, la longueur non filetée au fond des trous borgnes. Par ailleurs, le couple nécessaire lors d'une opération de filetage à la fraise est inférieur à celui en taraudage. Par contre, le temps de coupe est généralement bien plus court en taraudage. Pour ces raisons, la technique du filetage à la fraise est bien adaptée et utilisée pour la fabrication de pièces à forte valeur ajoutée, et à l'obtention de filet de grand diamètre.

Les problématiques associées à la technique du filetage à la fraise sont diverses. Comme pour toutes les opérations d'usinage avec des outils de forme (fraisage ou rectification), il se pose la question des interférences géométriques et la précision des surfaces obtenues [1]. La seconde problématique est la modélisation des efforts de coupe. Il existe des approches sur ce thème [2] mais la complexité du filetage à la fraise fait qu'actuellement les approches utilisées sont simplifiées par l'absence de prise en compte de :

- la définition de l'arête de la fraise et de l'angle de coupe;

- du problème 3D pour le calcul de la section coupée : assimilation à une opération de fraisage rectiligne [2] ;

- la géométrie locale de la coupe lors de la modélisation d'effort.

Cette étude propose de formaliser une approche précise et simplifiée de la situation géométrique du filetage à la fraise.

Le cas traité dans cette étude portera sur la réalisation d'un filet métrique M16 × 2 à droite avec une fraise à fileter de diamètre $12 \mathrm{~mm}$. Les notations utilisées pour le filet sont celles définies par la norme ISO 68-1 [3]. Le tableau récapitule toutes les données communes aux applications. La modélisation géométrique est faite sur un pas, le paramètre $a_{z}$ varie donc de 0 au pas $P$.

\section{Modélisation géométrique de la fraise}

Cette partie de l'étude traite successivement de la définition d'un profil de fraise, et de la modélisation de l'arête de coupe, en lien avec celle de la face de coupe, puis de la face en dépouille. Ces modélisations géométriques examinent deux hypothèses pour l'arête, pour finalement ne considérer que celle qui se rapproche le plus de l'affutage réel des fraises à fileter.

\subsection{Profil de la fraise}

Le profil d'une fraise (PF) est coïncidant avec le profil intérieur du filet à générer. Ce profil de fraise est composé de segments de droite, passant par les points $\mathbf{P}_{\mathrm{fi}}$ comme le 
Tableau 1. Données de l'étude.

\begin{tabular}{ccc|ccc}
\hline \multicolumn{3}{c|}{ Filet M16 $\times 2$} & \multicolumn{3}{c}{ Fraise à fileter } \\
\hline$D$ & $16 \mathrm{~mm}$ & Cf. Figure 8 & $D_{\mathrm{f}}$ & $12 \mathrm{~mm}$ & Cf. Figure 2 \\
$P$ & $2 \mathrm{~mm}$ & & $P$ & $2 \mathrm{~mm}$ & Cf. Figure 2 \\
$D_{1}$ & $13,93 \mathrm{~mm}$ & Cf. Figure 8 & $D_{2 \mathrm{f}}$ & $10,70 \mathrm{~mm}$ & Cf. Figure 2 \\
$D_{2}$ & $14,70 \mathrm{~mm}$ & Cf. Figure 8 & Z & 5 dents & \\
\hline
\end{tabular}

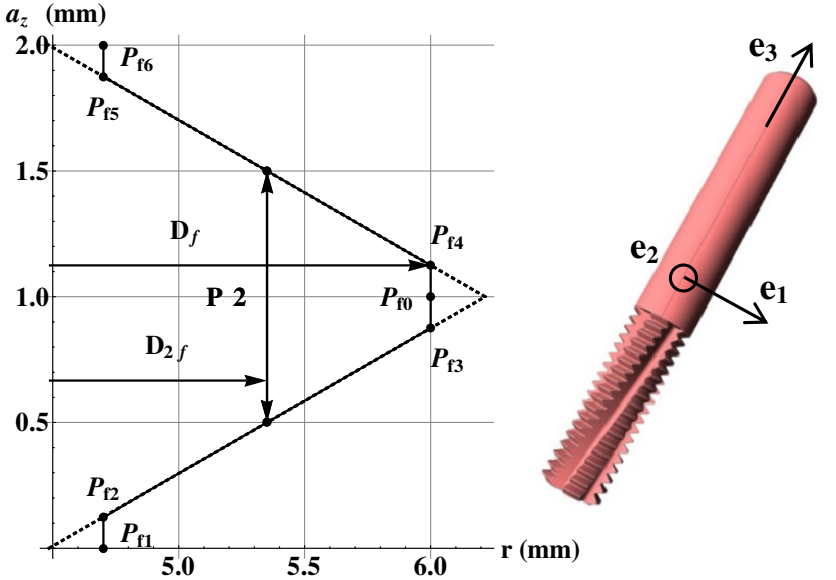

Fig. 2. Profil de la fraise à fileter dans le repère outil.

montre la figure 2. Il peut être paramétré, cf. équation (1), fonction de l'altitude $a_{z}$ d'un point de la fraise dans le repère de l'outil $\left(o, \mathbf{e}_{1}, \mathbf{e}_{3}\right)$. La fonction $\mathrm{PF}_{\mathrm{r}}$ est donc une fonction linéaire continue par morceaux telle que celle définie dans [1].

$$
\mathbf{P F}\left(a_{z}\right)=\left[\operatorname{PF}_{\mathrm{r}}\left(a_{z}\right), a_{z}\right]^{T}
$$

\subsection{Arête de coupe sans définition de la face de coupe : cas 1}

Dans le cas d'une fraise cylindrique, l'arête de coupe est généralement définie par une hélice circulaire. Si cette approche est appliquée à la fraise à fileter, alors la position angulaire d'un point de l'arête de coupe est liée à l'altitude $a_{z}$, par l'angle d'hélice des goujures $\lambda_{\mathrm{sf}}$, et le rayon de la fraise $\mathrm{PF}_{\mathrm{r}}$, suivant l'équation :

$$
\theta_{\mathrm{ac} 1}\left(a_{z}\right)=a_{z} \cdot \tan \left(\lambda_{\mathrm{sf}}\right) / \mathrm{PF}_{\mathrm{r}}\left(a_{z}\right)
$$

En conséquence, l'arête de coupe $\mathbf{A C}_{1}$ peut être paramétrée en fonction du profil de la fraise $\mathrm{PF}_{\mathrm{r}}$, par l'équation (3) dans un repère de l'outil $\left(o, \mathbf{e}_{1}, \mathbf{e}_{2}, \mathbf{e}_{3}\right)$. Cette formulation est rapide et simple; toutefois elle ne prend pas en compte le fait que la face de coupe de la fraise est aussi inclinée dans la direction radiale à cause de l'angle de coupe orthogonal.

$$
\mathbf{A C}_{1}\left(a_{z}\right)=\left[\mathrm{PF}_{\mathrm{r}}\left(a_{z}\right) \cdot \cos \left(\theta_{\mathrm{ac} 1}\right), \mathrm{PF}_{\mathrm{r}}\left(a_{z}\right) \cdot \sin \left(\theta_{\mathrm{ac} 1}\right), a_{z}\right]^{T}
$$

\subsection{Arête de coupe avec définition de la face de coupe : cas 2}

La seconde approche pour définir l'arête de coupe $\mathbf{A C}_{2}$, considère l'angle d'hélice de la goujure $\lambda_{\mathrm{sf}}$ ainsi que l'angle de coupe orthogonal $\gamma_{\text {of }}$ le long de l'arête de coupe frontale. Cette approche nécessite de définir formellement la face de coupe $\mathbf{F C}$, et l'hypothèse retenue est d'associer un hélicoïde à la forme de la goujure. Cette hypothèse vient de la connaissance de la technique d'affutage utilisée et a fait l'objet d'une vérification suite à la numérisation d'un outil. Dans le cas d'outil de petit diamètre, du fait d'interférence de taillage la face de coupe n'est plus assimilable à un hélicoïde.

La démarche adoptée dans cette étude pour modéliser la goujure, est celle explicitée dans [4]. L'hélicoïde est une courbe réglée dont la courbe guide est une hélice circulaire de diamètre égal à celui de la fraise et d'angle d'hélice celui de la goujure $\lambda_{\text {sf }}$. La règle est inclinée de l'angle de coupe orthogonal $\gamma_{\text {of }}$ par rapport à la direction radiale.

Dès lors l'arête de coupe $\mathbf{A C}_{2}$ est définie comme étant la courbe qui se situe sur la face de coupe $\mathbf{F C}\left(a_{z}, r\right)$ et qui respecte le profil de la fraise PF. Mathématiquement, l'arête de coupe $\mathbf{A C}_{2}$ est obtenue par la convolution des deux fonctions :

$$
\mathbf{A C}_{2}\left(a_{z}\right)=\mathbf{F C}\left(a_{z}, \operatorname{PF}_{\mathrm{r}}\left(a_{z}\right)\right)
$$

L'arête ainsi définie, la position angulaire d'un point se calcule par l'équation :

$$
\theta_{\mathrm{ac} 2}\left(a_{z}\right)=\arctan \left(\mathbf{A C}_{2}\left(a_{z}\right) \cdot \mathbf{e}_{1}, \mathbf{A} \mathbf{C}_{2}\left(a_{z}\right) \cdot \mathbf{e}_{2}\right)
$$

La figure 3 présente la comparaison des deux arêtes de coupe obtenues ainsi que le profil de la fraise. Le long de l'arête de coupe frontale (acf), les deux formulations sont identiques, puisque dans cette zone l'arête de coupe est bien une hélice circulaire. En dehors de cette zone, l'arête de coupe $\mathbf{A C}_{2}$ qui prend en compte l'angle de coupe est en retrait par rapport à l'arête de coupe $\mathbf{A C}_{1}$, du fait d'un angle de coupe $\gamma_{\text {of }}$ positif. C'est sur l'arête de coupe inférieure (aci) qu'il existe un écart maximal entre les deux formulations, dans ce cas, il se caractérise, dans notre cas d'étude, par un écart angulaire d'environ $4^{\circ}$.

\subsection{Face de dépouille}

Les méthodes d'affûtage des faces en dépouille d'une fraise se caractérisent en général par un angle de dépouille qui est constant dans la direction radiale de l'outil, i.e. l'angle de dépouille en travail $\alpha_{\mathrm{ff}}$ constant. La forme de 

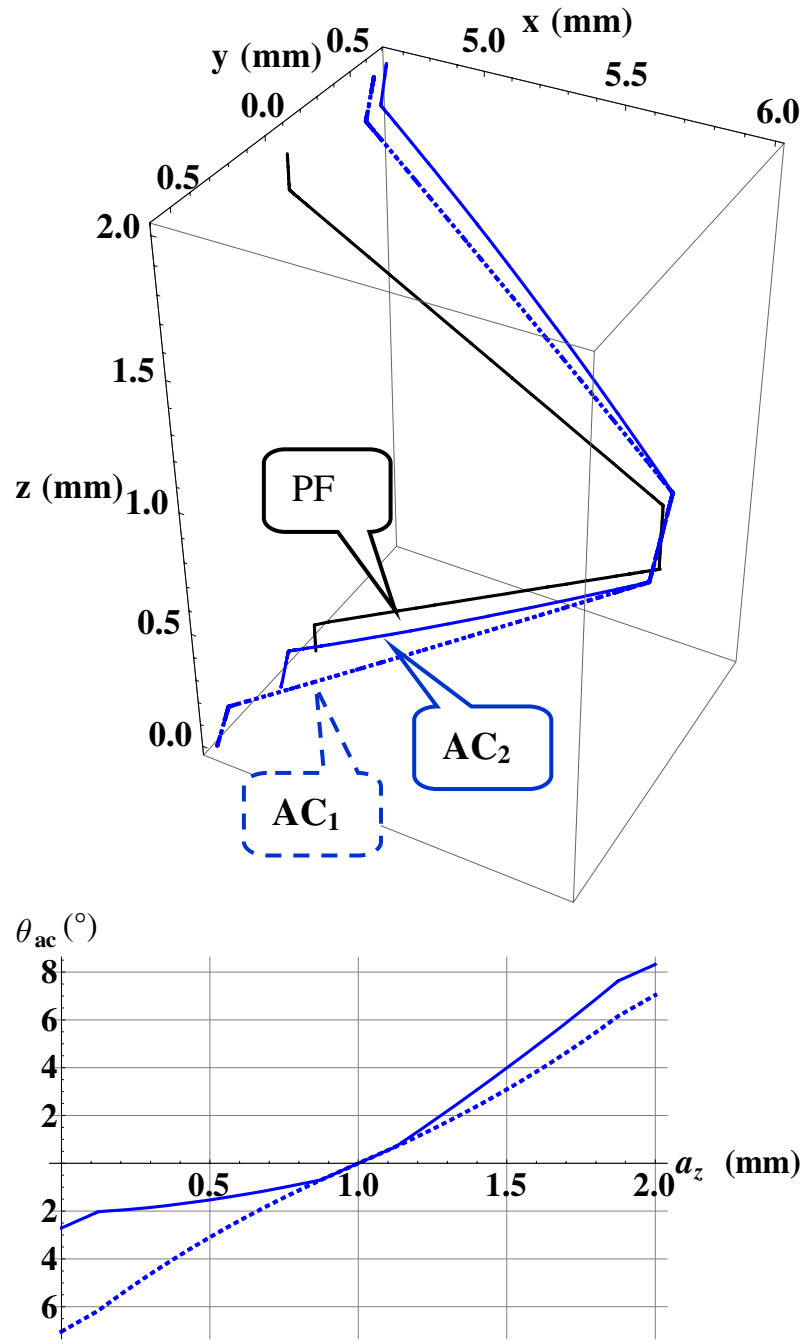

Fig. 3. Comparaison des deux définitions de l'arête de coupe $\left(\gamma_{\mathrm{of}}=10^{\circ} ; \lambda_{\mathrm{sf}}=30^{\circ}\right)$.

cette surface de dépouille peut varier; toutefois, ce qui est important, c'est sa tangente à l'arête de coupe, qui permet de définir l'angle de dépouille. L'hypothèse retenue, pour la modélisation de la face en dépouille est que dans chaque section radiale, la face de dépouille est un arc de cercle de rayon $R_{\mathrm{FD}}$, comme le montre la figure 4 . Ainsi le centre de la section CFD est ensuite calculé :

$$
\begin{aligned}
\mathbf{C F D}\left(a_{z}\right)=\operatorname{AC}\left(a_{z}\right)+ & R_{\mathrm{FD}} \cdot \cos \left(\alpha_{\mathrm{ff}}\right) \cdot \operatorname{VR}\left(a_{z}\right) \\
& +R_{\mathrm{FD}} \cdot \sin \left(\alpha_{\mathrm{ff}}\right) \cdot \operatorname{VNR}\left(a_{z}\right)
\end{aligned}
$$

fonction de l'angle de dépouille en travail souhaité le long de l'arête. La face en dépouille peut ainsi être paramétrée par l'équation :

$$
\begin{array}{r}
\mathbf{F D}\left(a_{z}, \theta\right)=\mathbf{C F D}\left(a_{z}\right)+R_{\mathrm{FD}}\left[\cos \left(\theta+\alpha_{\mathrm{ff}}+\theta_{\mathrm{ac}}\right),\right. \\
\left.\sin \left(\theta+\alpha_{\mathrm{ff}}+\theta_{\mathrm{ac}}\right), 0\right]^{T}
\end{array}
$$

La figure 5 montre la modélisation géométrique de l'arête de coupe $\left(\mathbf{A C}_{2}\right)$, de la face de coupe $(\mathbf{F C})$ et de la face en dépouille (FD) d'une fraise à fileter. D'autre part une

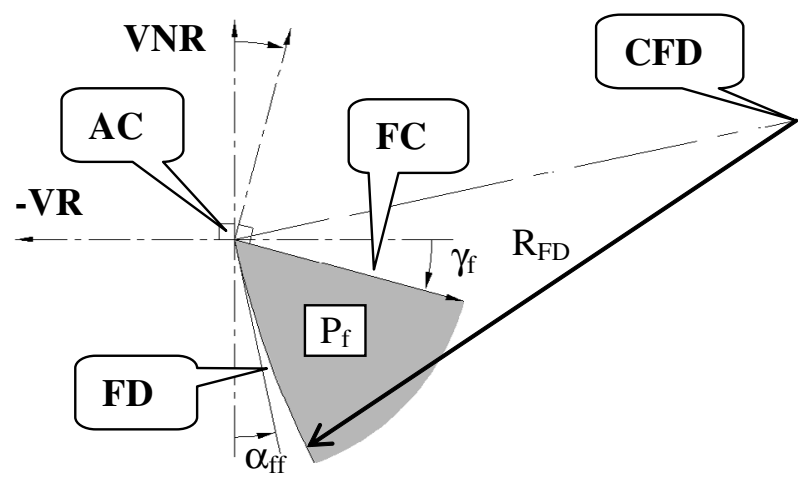

Fig. 4. Modélisation de la face en dépouille $\left(\gamma_{\mathrm{of}}=10^{\circ} ; \lambda_{\mathrm{sf}}=\right.$ $30^{\circ}$ ).

fraise a été numérisée afin d'obtenir un nuage de points de sa surface. La correspondance des points de mesure avec le modèle géométrique, au niveau des parties actives de l'outil, est bonne, et valide donc les hypothèses du modèle géométrique.

\section{Angles de la fraise à fileter}

Les définitions utilisées sont celles de la norme ISO 3002-1 [5]. Sur la base des surfaces de coupe et de dépouille paramétrées précédemment, il est possible de définir les normales à ces surfaces et les intersections avec les plans de coupe tels que le plan normal $\mathbf{P}_{\mathrm{n}}$ à l'arête et le plan orthogonal $\mathbf{P}_{\mathrm{o}}$ de l'outil. Les calculs vectoriels sont conduits comme dans l'étude [4]. Cela permet ensuite de déterminer les angles de coupe et de dépouille réels le long de l'arête de coupe, sachant que la définition des surfaces repose :

- pour la face de coupe sur l'angle d'hélice $\left(\lambda_{\mathrm{sf}}\right)$ et l'angle de coupe orthogonal $\left(\gamma_{\text {of }}\right)$ sur l'arête frontale;

- pour la face en dépouille sur un angle de dépouille en travail $\left(\alpha_{\mathrm{ff}}\right)$ constant.

\subsection{Angle de coupe}

Il est choisi d'analyser l'angle de coupe normal, car il est pris dans le plan de coupe normal qui est le plus proche du plan d'écoulement du copeau sur la face de coupe. Les angles de coupe pris dans le plan de travail $\mathbf{P}_{\mathrm{f}}$ ou le plan orthogonal $\mathbf{P}_{\mathrm{o}}$ ne sont absolument pas significatifs de ce que la matière voit pendant la coupe.

La figure 6 montre que l'angle de coupe normal local $\left(\gamma_{\mathrm{n}}\right)$ varie considérablement le long de l'arête. L'augmentation de l'angle d'hélice de goujure $\left(\lambda_{\mathrm{sf}}\right)$, tend à introduire de la coupe fortement négative sur l'arête de coupe supérieure (acs) et l'inverse sur l'arête de coupe inférieure (aci). Cela a pour conséquence d'induire localement sur l'arête des efforts de coupe très différents. 


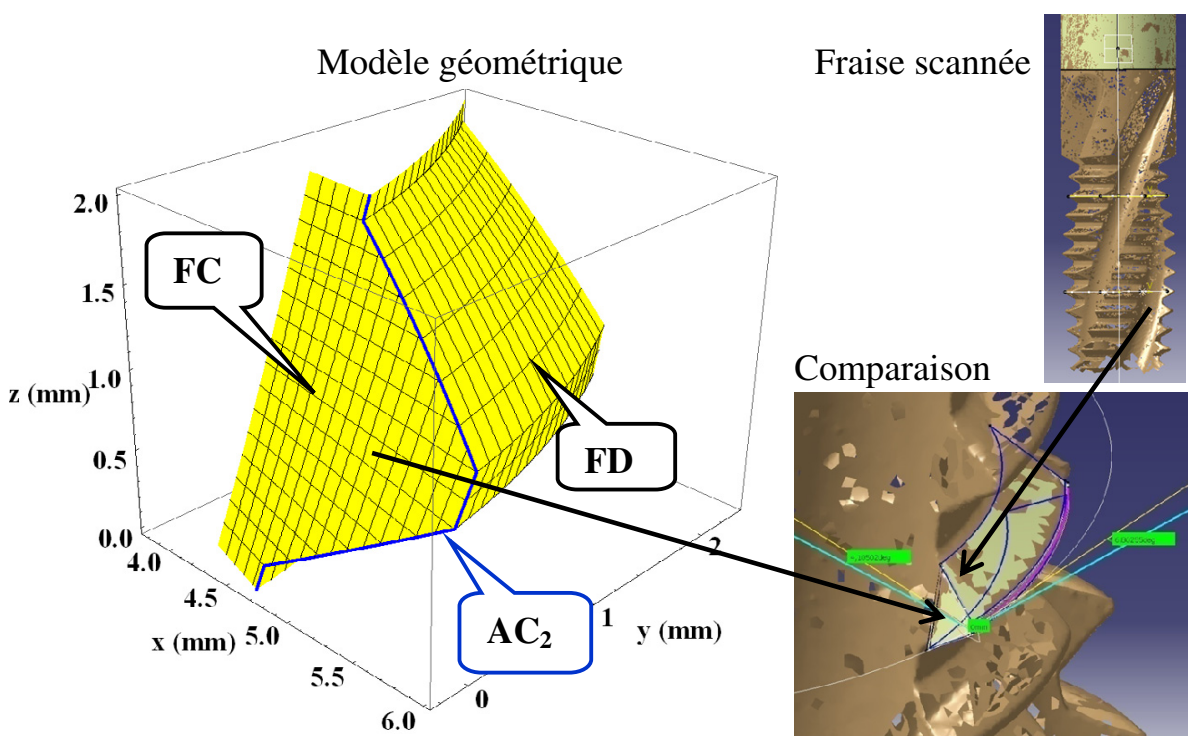

Fig. 5. Modélisation géométrique d'une fraise à fileter et comparaison avec une surface numérisée $\left(\gamma_{\mathrm{of}}=10^{\circ} ; \lambda_{\mathrm{sf}}=30^{\circ}\right.$; $\left.\alpha_{\mathrm{ff}}=10^{\circ}\right)$.

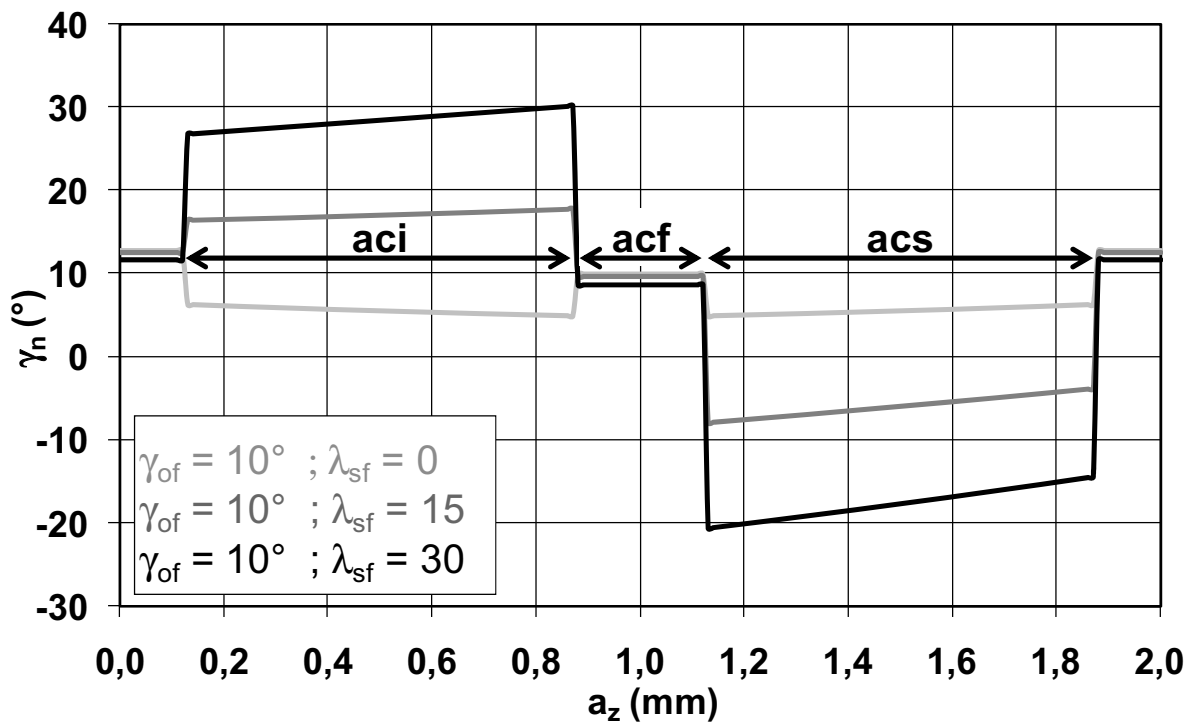

Fig. 6. Variation de l'angle de coupe normal le long de l'arête de coupe.

\subsection{Angle de dépouille}

Lors d'une opération d'usinage, il existe un contact entre la face en dépouille de l'outil et la pièce, dont il résulte des efforts. Le plan de coupe $\mathbf{P}_{\mathrm{o}}$, par définition, contient à la fois la normale à ce contact et la vitesse de coupe, qui est la vitesse de glissement au contact. De fait, les efforts liés au frottement de la face en dépouille de la fraise sont liés à l'angle de dépouille orthogonal local $\left(\alpha_{\mathrm{o}}\right)$.

De la même manière que pour l'angle de coupe normal, l'angle de dépouille orthogonal $\left(\alpha_{\mathrm{o}}\right)$ est donc analysé le long de l'arête de coupe. Comme le montre la figure 7, sur les arêtes de coupe des flancs, cet angle a des valeurs environ deux fois inférieures à celles sur l'arête de coupe frontale (acf). En conséquence, les efforts liés au frottement de la face en dépouille de la fraise seront affectés par cette propriété géométrique.

\section{Calcul de la section coupée}

Le calcul des sections coupées en fraisage est indispensable pour la modélisation des efforts et la compréhension de la technique d'usinage. Pour une fraise cylindrique ou une fraise boule, il existe principalement trois approches pour mener ce calcul :

- Considérer directement que l'épaisseur coupée $h$ varie suivant la loi $h=f_{\mathrm{z}} \sin \theta$, où $\theta$ est la position angulaire de la fraise [2].

- Assimiler la trajectoire des dents de la fraise à des cercles et calculer la distance normale entre eux [6]. 


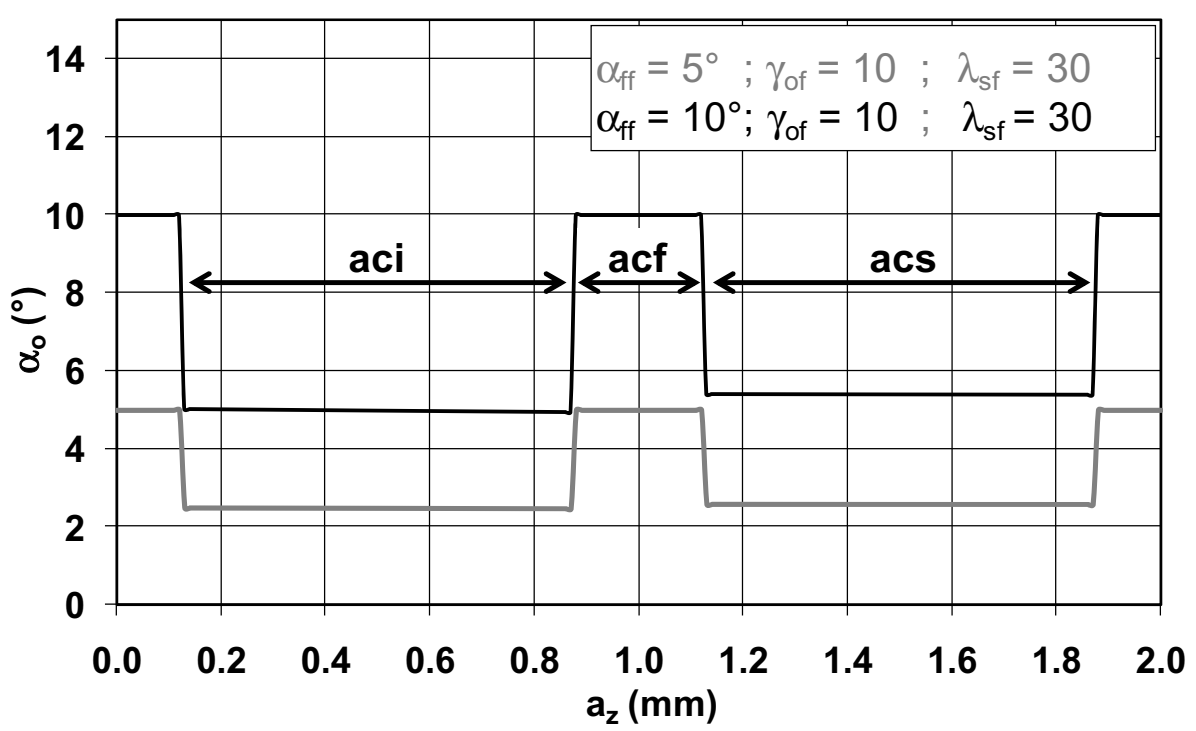

Fig. 7. Variation de l'angle de dépouille normal le long de l'arête de coupe.

- Définir la trajectoire réelle des dents par des cycloïdes et calculer la distance normale entre elles $[7,8]$.

A priori, cette dernière est la solution exacte et a déjà été mise en œuvre dans le cas de la fraise à fileter [9]. Elle s'avère lourde en temps de calcul, car elle repose sur la détermination des distances normales entre les surfaces 3D balayées par les dents de la fraise. Il est donc développé ci-dessous la méthode reposant sur des cercles, tout en ne simplifiant pas le problème à un cas plan et en prenant en compte les spécificités de la configuration du filetage à la fraise.

\subsection{Trajectoire d'une fraise à fileter}

Lors d'une opération de filetage à la fraise, la trajectoire du centre fraise (TCF) est une hélice circulaire, et la vitesse d'avance programmée est appliquée par la commande numérique dans l'espace le long de cette courbe. Elle peut être paramétrée, cf. [1], par les équations :

$$
\Omega=Z \cdot f_{z} \cdot \omega /\left(2 \pi \cdot \sqrt{p^{2}+R_{\mathrm{tcf}}^{2}}\right)
$$

$$
\mathbf{T C F}(\mathrm{t})=\left[R_{\mathrm{tcf}} \cdot \cos (\Omega . t), R_{\mathrm{tcf}} \cdot \sin (\Omega . t), p . \Omega . t\right]^{T}
$$

dans le repère lié à la pièce $\left(O, \mathbf{E}_{1}, \mathbf{E}_{2}, \mathbf{E}_{3}\right)$. Les grandeurs $R_{\mathrm{tcf}}, p, \omega, \Omega$ représentent respectivement, le rayon de l'interpolation hélicoïdale, le pas par radian, la vitesse angulaire de la fraise et la vitesse angulaire de l'axe de la fraise.

La projection dans le plan $\left(\mathbf{E}_{1}, \mathbf{E}_{2}\right)$ de l'avance $f_{z}$, est donnée par l'équation :

$$
f_{z 12}=f_{z}\left(1+\left(\frac{p}{R_{\mathrm{tcf}}}\right)^{2}\right)^{-1}
$$

et la composante verticale de l'avance est donc obtenue par la relation :

$$
f_{z 3}=\sqrt{f_{z}^{2}-f_{z 12}^{2}}
$$

\subsection{Calcul de la section coupée : méthode des cercles}

La figure 8 représente les hypothèses pour le calcul de l'épaisseur coupée $h$, le long de l'arête (fonction de $a_{z}$ ) et pour différentes positions angulaires de la fraise (fonction de $\theta$ ). La trajectoire des dents est approximée par des cercles de rayon défini par le profil de la fraise $(\mathrm{PF})$. La distance suivant un arc de cercle $\left(\mathrm{C}_{1} \mathrm{C}_{2}\right)$ entre les centres des deux cercles, représentant la trajectoire de deux dents consécutives, est égale à l'avance projetée dans le plan $f_{z 12}$. Afin de prendre en compte le mouvement vertical de la fraise dans la détermination de la section coupée, le rayon des deux cercles retenus correspond sur le profil de la fraise PF à un point d'altitude $a_{z}$ donné et à un point d'altitude $a_{z}-f_{z 3}$. L'épaisseur coupée $h$ est ensuite calculée comme étant la distance normale du cercle de centre $\mathrm{C}_{2}$ au cercle de centre $\mathrm{C}_{1}$, pondéré par le sinus de l'angle de direction d'arête $\left(\kappa_{\mathrm{r}}\right)$. Ce dernier est lié au profil de la fraise donc à celui du filet, et vaut respectivement $30^{\circ}, 90^{\circ}$ et $150^{\circ}$ sur l'arête de coupe inferieure, frontale et supérieure d'une fraise pour l'usinage d'un filet ISO métrique.

La figure 9 présente le résultat d'un calcul de section coupée exécuté avec cette méthode des cercles. Les résultats montrent que l'épaisseur coupée $h$ diminue en fonction du temps, i.e. de l'angle $\theta$, il s'agit donc d'un usinage en avalant. L'arête frontale coupe une épaisseur plus grande que celles des flancs. Ceci s'explique par le fait qu'il s'agit du fraisage en avalant d'un filet avec un pas à droite. La composante verticale de la vitesse est donc ascendante. Il en résulte que l'arête de coupe supérieure (acs) est face à la direction de l'avance axiale, et donc que 


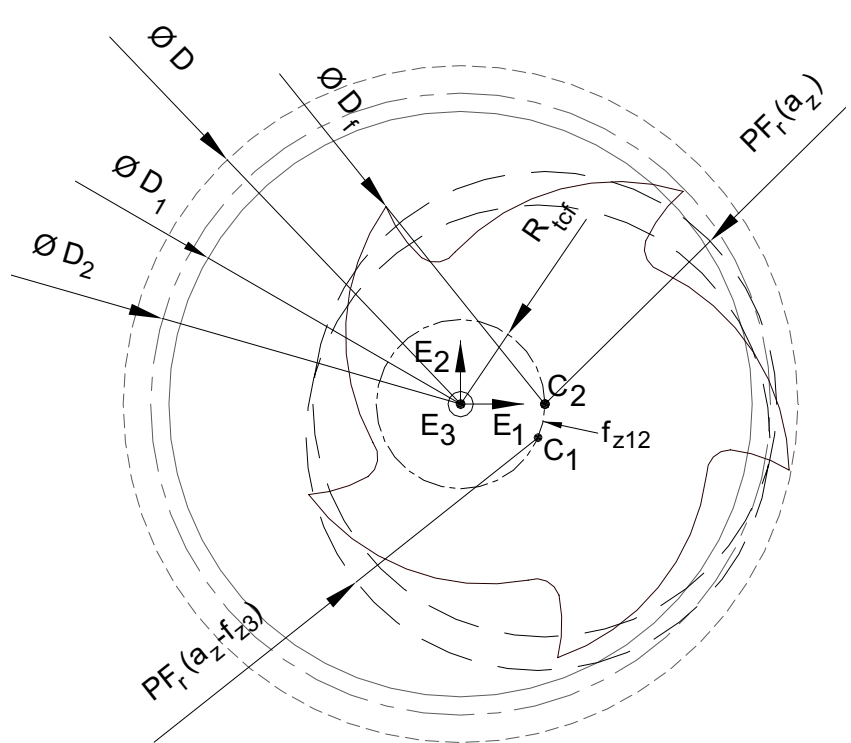

Fig. 8. Configuration pour le calcul de l'épaisseur coupée $h$.

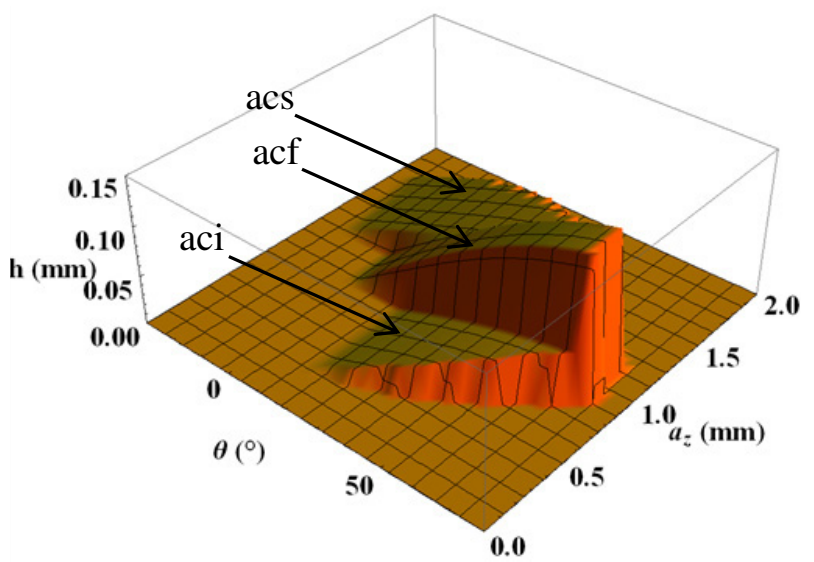

Fig. 9. Épaisseur coupée $h$ le long de l'arête $\left(\gamma_{\text {of }}=10^{\circ}, \lambda_{\mathrm{sf}}=\right.$ $\left.30^{\circ}, f_{\mathrm{z}}=0,15 \mathrm{~mm} \cdot \mathrm{tr}^{-1} \cdot \mathrm{dt}^{-1}\right)$.

l'épaisseur coupée est plus importante que celle coupée par l'arête de coupe inférieure (aci). C'est l'inverse dans le cas d'un usinage en opposition ou dans le cas du fraisage d'un filet avec un pas à gauche.

Ce cas a déjà étudié avec la méthode exacte [9]. Il est donc possible de comparer la méthode des cercles présentée ici, avec la méthode reposant sur la distante normale entre les surfaces balayées réellement par les dents. La figure 10 présente l'erreur absolue (EA) entre ces deux méthodes. L'erreur commise par la méthode des cercles est maximale pour les faibles sections coupées sans toutefois dépasser $10 \mu \mathrm{m}$ dans le cas étudié. D'autre part, la méthode des cercles tend vers la solution exacte dans la section coupée maximale, donc elle n'engendre pas d'erreur pour la détermination des efforts de coupe les plus importants.

Cette nouvelle approche nécessite peu de ressource de calcul et permet de bien reproduire le problème 3D. Cela rend réalisable une approche d'identification inverse d'un modèle d'efforts de coupe en filetage à la fraise.

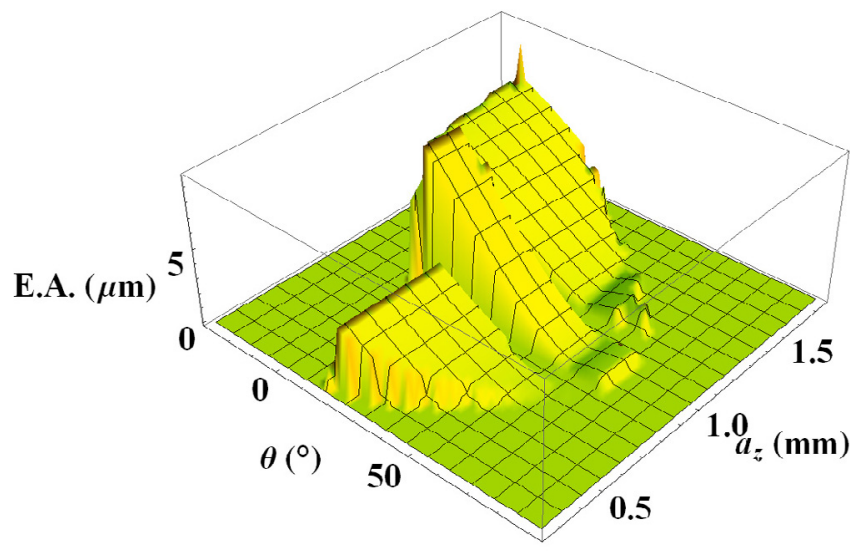

Fig. 10. Erreur absolue sur le calcul de l'épaisseur coupée avec la méthode des cercles $\left(\gamma_{\mathrm{of}}=10^{\circ}, \lambda_{\mathrm{sf}}=30^{\circ}, f_{\mathrm{z}}=\right.$ $\left.0,15 \mathrm{~mm} \cdot \mathrm{tr}^{-1} \cdot \mathrm{dt}{ }^{-1}\right)$.

\section{Analyse et conclusion}

Ces travaux montrent des particularités importantes des opérations de filetage à la fraise. La modélisation géométrique proposée porte sur les points suivants :

- Modélisation géométrique de la surface de coupe et de dépouille de l'outil et détermination des angles de coupe.

- Définition de la trajectoire du centre outil et calcul de la section coupée.

Les angles de coupe et de dépouille varient considérablement le long de l'arête de coupe. L'angle de coupe normal peut devenir négatif sur le flanc supérieur du fait de l'angle d'hélice de la goujure. Quant à l'angle de dépouille orthogonal, il est simplement divisé par deux sur les arêtes des flancs et cela du fait de l'orientation de l'arête de coupe, donc du profil du filet à usiner.

L'épaisseur de la section coupée est variable le long de l'arête, et lors d'un usinage en avalant le flanc du haut est plus chargé que le flanc du bas. Or il se trouve que l'angle de coupe normal est négatif sur le flanc du haut, ce qui est donc défavorable. Dans le cas d'usinage en opposition, c'est le flanc du bas qui serait le plus chargé ce qui paraît donc plus profitable.

L'angle d'hélice de la goujure introduit de la coupe négative ce qui augmente les efforts de coupe. Toutefois, cet angle a l'intérêt de répartir dans le temps la section coupée, ce qui diminue les pics des efforts de coupe. De cette analyse qualitative, il faut déduire qu'il devrait donc exister une valeur optimale de l'angle de goujure en terme de variation et de pic d'efforts de coupe. Cette optimisation doit être possible avec un modèle adapté de la pression de coupe.

En conclusion, une modélisation mécanique des efforts de coupe, pour une opération de filetage à la fraise, doit prendre en compte les paramètres locaux de la géométrie de l'arête ainsi que ses conditions de chargement. Cela nécessite notamment d'établir que l'énergie spécifique de coupe considérée n'est pas seulement fonction de 
l'épaisseur coupée mais aussi fonction de la géométrie de coupe locale.

\section{Références}

[1] G. Fromentin, G. Poulachon, Modeling of interferences during thread milling operation, Int. J. Adv. Manuf. Technol. 49 (2010) 41-51

[2] A.C. Araujo, J.L. Silveira, M.B.G. Jun, S.G. Kapoor, R. Devor, A model for thread milling cutting forces, Int. J. Machine Tools Manuf. 46 (2006) 2057-2065

[3] ISO 68-1:1998 standard, ISO general purpose screw threads, Basic profile, Part 1: Metric screw threads

[4] G. Fromentin, G. Poulachon, Geometrical analysis of thread milling, Part 1: Evaluation of tool angles, Int. J. Adv. Manuf. Technol. 49 (2010) 73-80
[5] ISO 3002-1 standard, Basic quantities in cutting and grinding. Part 1: Geometry of the active part of cutting tools, General terms, reference systems, tool and working angles

[6] Martin B.G. Jun, Anna Carla Araujo, Modeling and analysis of the thread milling operation in the combined drilling/thread milling process, Proceedings of the 2008 International Manufacturing Science and Engineering Conference, MSEC2008, October 7-10, 2008, Evanston, Illinois, USA

[7] L. Saï, W. Bouzid, A. Zghal, Chip thickness analysis for different tool motions: for adaptive feed rate, J. Mater. Proc. Technol. 204 (2008) 213-220

[8] X.-G. Liang, Z.-Q. Yao, An accuracy algorithm for chip thickness modeling in 5-axis ball-end finish milling, Computer-Aided Design 43 (2011) 971-978

[9] G. Fromentin, G. Poulachon, Geometrical analysis of thread milling, Part 2: Calculation of uncut chip thickness, Int. J. Adv. Manuf. Technol. 49 (2010) 81-87 